\title{
Compensation of Industrial CMOS Camera and Image Enhancement for Sounding Rocket Astrophysical Experiments
}

\author{
1) Ivo Vertat, ${ }^{2)}$ Pavel Fiala \\ Faculty of Electrical Engineering \\ University of West Bohemia \\ Pilsen, Czech Republic \\ 1)ivertat@kae.zcu.cz, ${ }^{2)}$ pavelf@kae.zcu.cz
}

\begin{abstract}
Suborbital missions of sounding rockets along the parabolic trajectory are commonly used for the Earth's near space environment research or for the astrophysical observations with the time duration only in the order of several minutes. Such short-term missions are usually performed with a low-cost equipment as the exact opposite of long-term satellite missions with high-end and expensive technologies. In this paper there is described the usage of a low-cost industrial CMOS camera for the confirmation of accurate pointing of the Roentgen telescope during the Vela supernova observation. It requires a robust method of the image post-processing and the compensation of real properties of the camera system faced to the extreme space environment. Also a specific mode of the image acquisition is required due to temperature changes during the suborbital mission. As the result of our work, a low-cost camera could be used for simple and short-term tasks in space instead of an expensive space qualified system.
\end{abstract}

Keywords - suborbital mission; sounding rocket; camera system for space; image enhancement; camera properties compensation; lobster eye Roentgen telescope

\section{INTRODUCTION}

Traditional space qualified camera systems are expensive, because the high quality images and a long-term reliability in the space environment are required. These requirements have to be ensured by many technically complicated precautions including an active thermal management, using of the radiation hardened electronics parts with a wide range of operational temperatures, adding the mechanical iris or special optical filters to prevent the thermal destruction of an image sensor by the direct Sun irradiation, etc. As a result, even the small space qualified camera system for CubeSat usually costs several thousands of euros. Such high costs are unjustifiable for low-cost and short-term missions of the sounding rockets.

One sounding rocket experiment was prepared by the Aerospace Research and Test Establishment in cooperation with the several other partners in the Czech Republic (Rigaku Innovative Technologies Europe, the Czech Technical University and the University of West Bohemia). The main purpose of this experiment is to test a new lobster eye Roentgen optics [1] with the pixel based particle detector during the observation of the Vela supernova pulsar. This experiment (called REX) is a secondary payload of the sounding rocket [2]. The primary telescope payload has a target with slightly different pointing. Therefore the REX experiment was extended by a visible light camera system for the confirmation of accurate pointing to the secondary target.

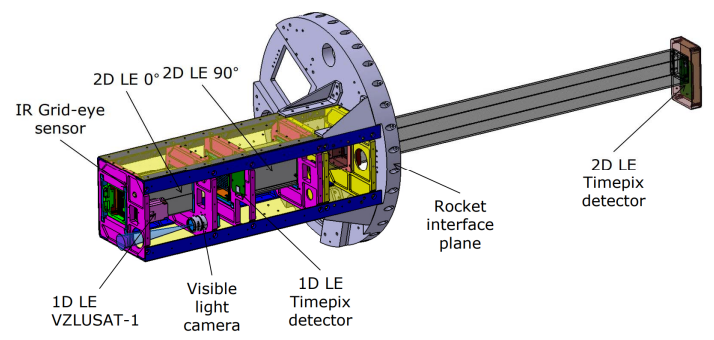

Figure 1. CAD model of REX experiment with two lobster eye Roetgen telescopes and with the camera system for visible light.

The camera system is based on the Ximea MQ013CG-E2 miniature USB-3 module with the $1 / 1.8$ " CMOS sensor size and the $1.3 \mathrm{Mpx}$ native resolution. It is equipped with the ThorLabs MVL50M23 lens (the focal length $50 \mathrm{~mm}$ ). The camera with lens has a field of view approximately $9.8^{\circ}$. This assembly offers all required properties in the total price less than 500 euros. The low power consumption and the aluminum camera case attached to the massive rocket mechanical structure mitigate the possibility of camera overheating in the vacuum conditions during the short-term mission. An absence of plastic materials in the camera body avoids the pollution of telescopes by material outgassing. The USB interface and the availability of drivers for Robot Operating System make easier implementation of the camera control software. A standard C-mount interface of the camera allows a large selection of the lens for the required field of view.
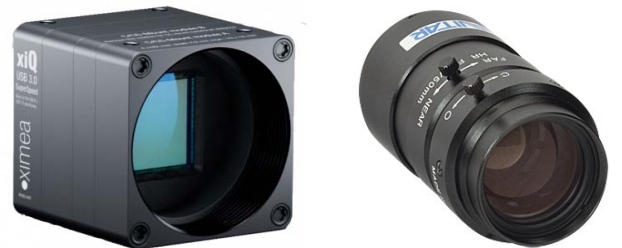

Figure 2. The camera system used in REX experiment for the confirmation of two Roentgen telescopes pointing toward the Vela supernova. 
However, the usage of common industrial cameras in a space star tracker requires a precise compensation of the real camera properties and at least some basic methods of the image enhancement. The camera properties compensation and the image enhancement are complicated by an unknown temperature profile to which the camera sensor will be exposed during the suborbital mission, because there will be no active thermal management for REX experiment.

The rest of this paper is organized as follows. An influence of the dark current and the thermal noise of image sensors is described in section II. Proposed methods of the image enhancement and closely related mode of image capturing are described in section III. Results of the ground tests with the capturing of stars, results of the proposed image enhancement applied on images from the ground test and the camera sensitivity are mentioned in section IV.

\section{THERMAL NOISE AND DARK CURRENT}

Common CMOS image sensors are very sensitive to the temperature changes due to the non-linear and non-uniform sensitivity of the pixels and the gains of their embedded amplifiers. The resulting image is affected by the dark current and thermal noise, especially during the image capturing in a poor light condition with a high gain of amplifiers. Such unprocessed and uncompensated images from the used Ximea camera are shown in fig. 3 for several different exposure settings.
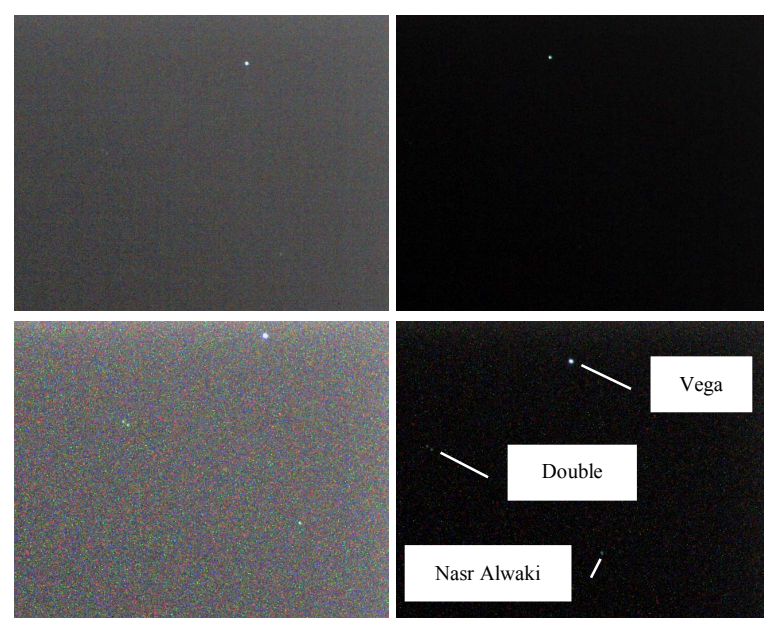

Figure 3. Original captured images during the ground testexposure time / sensor gain: $100 \mathrm{~ms} / 18 \mathrm{~dB}$ (left top), $100 \mathrm{~ms} / 5 \mathrm{~dB}$ (right top), $900 \mathrm{~ms} / 18 \mathrm{~dB}$ (left bottom), $900 \mathrm{~ms} / 5 \mathrm{~dB}$ (right bottom).

Increasing sensor gain and exposure time will allow more stars to be recognized in the image, but the dark current and thermal noise can cover the weak stars. The bright Vega star (magnitude 0) is well visible on all the images in fig. 3. The other three stars - Nasr Alwaki I (mag. 4.3), Double Double I (mag. 5), Double Double II (mag. 5.2) are visible in bottom images with longer exposure times. The other two weaker stars (mag. 6.5 and 6.85) are badly visible in the image with the longest exposure time and the highest gain (within the triangle created by the previously mentioned stars).
If the dark current and thermal noise remain uncompensated, the captured stars are poorly recognizable from their artefacts in the image. The REX experiment does not have an active thermal management, so it is expected to continually change the temperature of the sensor due to heating by its own consumption and also because of the temperature changes of the rocket body. Good recognition of weak stars around the Vela supernova will require precise image processing and enhancement based on the sensor's temperature knowledge and exposure settings.

\section{PROPOSED IMAGE ENHANCEMENT AND CAMERA MODE FOR IMAGE CAPTURING}

\section{A. Dark Current Compensation}

The dark current is the reverse bias leakage current in the individual photodiodes (pixels) of the image sensor. It is not caused by incident photons, but is caused by the random generation of electrons and holes in the depleted layer due to defects in the crystalline lattice. The dark current of CMOS sensors is deeper described in [3]. It depends on the sensor sensitivity, temperature and exposure time, but has a relatively static character. This means that the dark current is almost the same in the sequence of several images if all of sensor settings and temperature remain the same during the acquisition of images.

The dark current produces so-called fixed pattern noise with hot pixels. Hot pixels are areas in the image sensor where the dark current is abnormally high and the pixels in those areas have a high brightness even without incident light. Uncompensated fixed-pattern noise can mask weak stars in the image and unremoved hot pixels can be confused with stars. However, the dark current artefacts can be removed from the image by subtracting a dark frame. The dark frame is an image captured with the same exposure setting and at the same temperature of sensor, but with the closed iris. Better dark current compensation uses a series of dark frames and subtraction of the average dark frame. The averaging of dark frames suppresses the random thermal noise present in the images together with the dark current.

We used a method of subtracting an average dark frame in our suborbital experiment. The average dark frame is calculated from the sequence of dark frames captured during rocket flight with closed science section. As a backup solution, each image is stored with the exposure time, gain and temperature of the sensor for later shots of the dark frames in the laboratory conditions.

\section{B. Thermal Noise Supression}

The dark current compensation eliminates relatively static fixed pattern noise from the image and helps to enhance the recognition of stars in images captured with long exposure times and high gains. This effect of dark current compensation is shown in fig. 4, 5 and 6. However, the less bright stars could remain masked in the rest of the noise, mainly due to thermal noise. 
Typical methods for the noise suppression such as average filtering or median filtering over a sufficiently large area of adjacent pixels in the image could not be used in this case because the light of the weak stars has the same impulse character as the thermal noise. Image filtering over a small area does not suppress sufficiently thermal noise and filtering over a large area also suppresses the weak stars. However, the camera will be precisely pointed to the target for a few minutes and the time series of images with the same exposure setting will be captured during the observation. In this case, the thermal noise is random over the captured time series, but the light of stars is stationary. Filtration methods could thus be extended to the temporal dimension.

The selected noise suppression method uses a combination of spatial and temporal average filters. The spatial average filter only uses small $3 \times 3$ pixel area to maintain the point character of the stars, and the temporal average filter expands this area to 5 sequential images for better noise suppression. The result of dark current compensation, spatial/temporal filtering and gamma correction is shown in fig. 7 . Matlab software was used for this image processing.

\section{Camera Capturing Mode}

Reliable recognition of weaker stars in the image requires a long exposure time and high gain of the image sensor, but this setting also increases dark current and thermal noise, especially when the image sensor has a higher temperature. Since many aspects of the suborbital mission are not known in advance, we do not have any simulation of camera heating during a mission. The camera sensor temperature is expected to be between $30^{\circ} \mathrm{C}$ and $40^{\circ} \mathrm{C}$ during the mission. The forecast is based only on the functional test of the REX instrument panel assembly in the vacuum chamber.

Due to the uncertain camera temperature conditions during the mission, we propose a special camera capturing mode with changing exposure settings and idle intervals between the individual batches of image frames. The Odroid computer will start recording a batch of image frames as soon as the rocket turns on the power of the science section shortly before the launch. Each batch of image frames consists of several different exposure time settings (from $100 \mathrm{~ms}$ up to $900 \mathrm{~ms}$ ) and sensor gain settings (from $0 \mathrm{~dB}$ up to $18 \mathrm{~dB}$ ). A short idle time is placed between two batches to reduce heating of the image sensor. All image parameters (acquisition time, exposure time, sensor gain, sensor temperature) are also recorded.

This solution has several advantages. The dark frames for post-processing are captured before opening the telescope cover and also after closing it for all combinations of camera settings and at a temperature close to the real sensor temperature during star observation. Several different exposure settings minimize the risk of inappropriate camera settings due to unforeseen temperature conditions and could also be used for high dynamic range image postprocessing.

\section{CAMERA System GROUND TEST}

The proposed methods of dark current compensation and image enhancement were verified by capturing the Vega star with the surroundings of weaker stars. Vega is a bright and well recognizable star in the night sky, and a lot of weaker stars in the neighborhood are in the field of view to test the sensitivity of the camera. The direction of observation of the Vega star was also in the opposite direction from the light pollution from the nearby town of Pilsen.

One of the unprocessed images captured in the ground test is shown in fig. 4 . We recognize only stars like Vega (mag. 0), Nasr Alwaki I (mag. 4.3), Double Double I (mag. 5) and Double Double II (mag. 5.2). Other weaker stars are covered under dark current and thermal noise.

The dark current can be removed by dark frame compensation described in section III. The result of this compensation is shown in fig. 5 (the same brightness scaling as in fig. 4). After this compensation, additional stars may be recognized in the image, but thermal noise is still present in the image, as shown in fig. 6 (with gamma correction for better visibility).

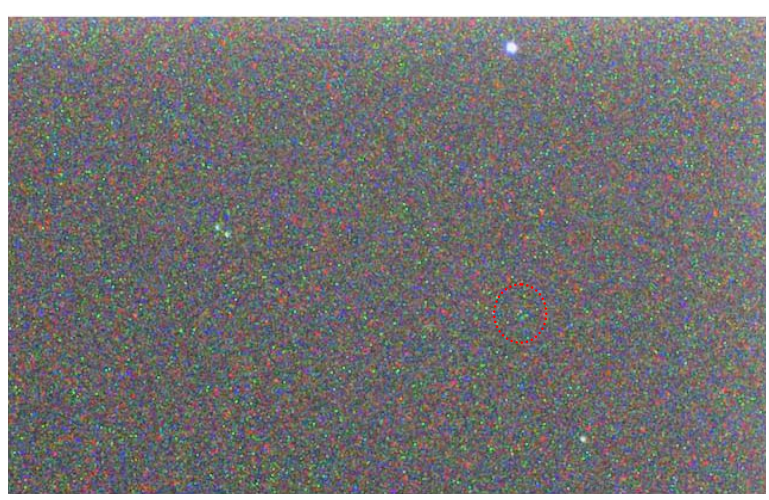

Figure 4. A cut from an unprocessed image captured during the ground test with an exposure time of $900 \mathrm{~ms}$ and sensor gain $18 \mathrm{~dB}$.

The red circle shows hot pixels caused by a dark current, easily interchangeable with stars.

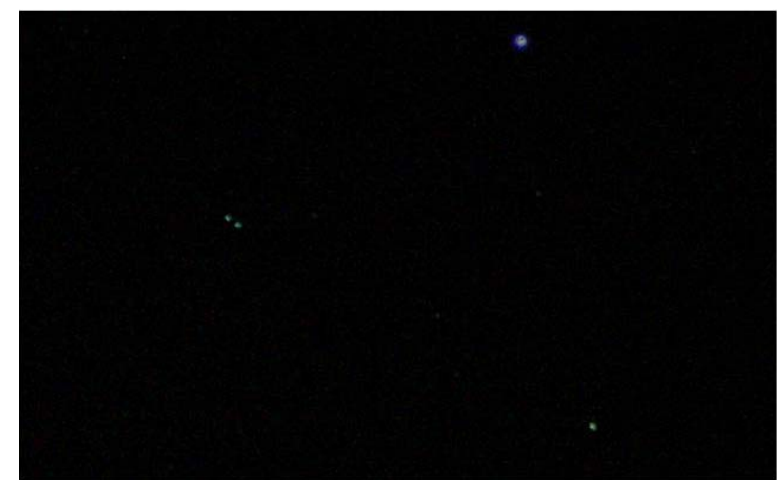

Figure 5. A cut from the processed image with dark frame compensation. Thermal noise and other recognizable stars are present in the image, but are not visible due to brightness scaling. 


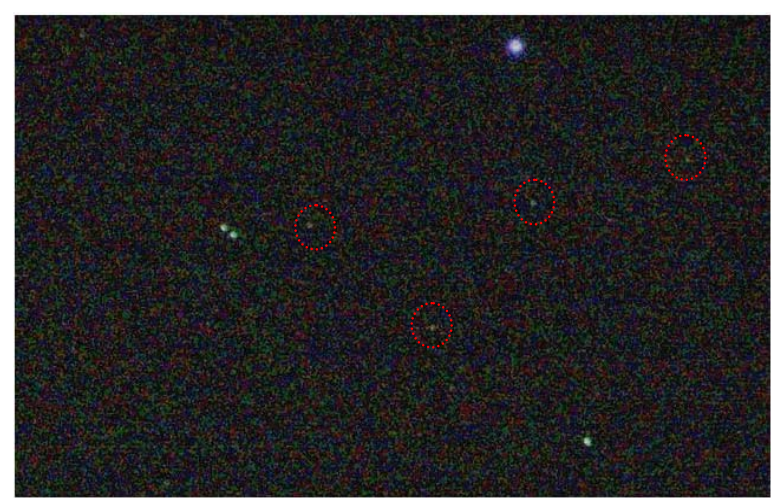

Figure 6. Gamma correction of previous image for better visualization of other recognized stars and residual thermal noise. Red circles show newly recognized stars.

At least four new stars are visible in the compensated image, e.g. HIP 91310 (mag. 7), HIP 91552 A (mag. 6.8), HIP 91820 (mag. 6.4), HIP 91898 (mag. 6.5) and several other areas are likely to be stars as well, but this is not entirely clear due to the thermal noise of the camera.

The thermal noise cannot be suppressed by the average filter only in spatial domain due to its similarity to the weak stars. However, it can be suppressed by a combination of spatial and temporal filtering, because the thermal noise is random in contrast to the static nature of the incident light of the stars. The result of spatial and temporal combined filtering is shown in fig. 7.

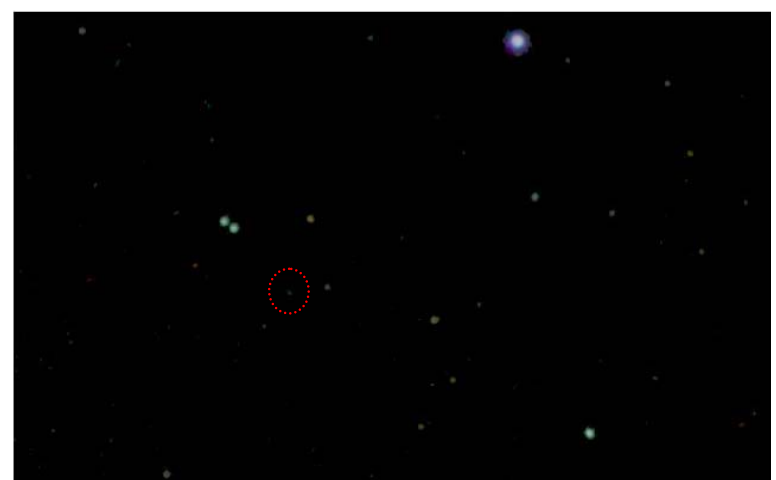

Figure 7. The result of image processing from Ximea camera during the ground test. Red circle shows the weakest star with magnitude 9.15 , recovered by post-processing from time series of captured images.

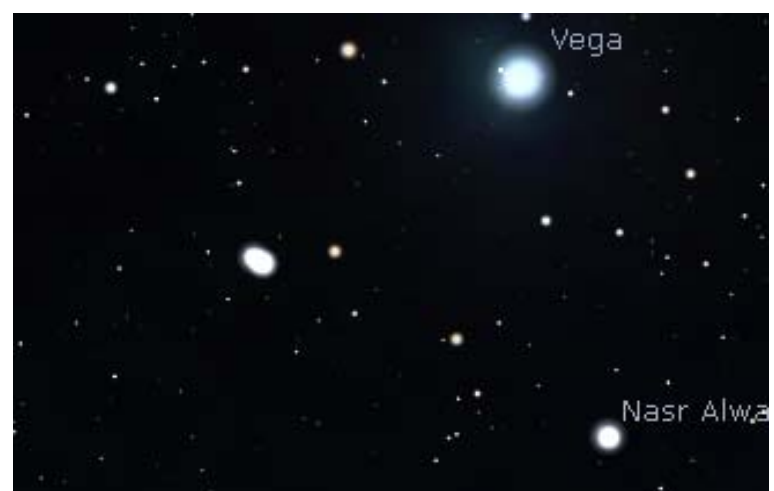

Figure 8. The sky map from Stellarium software with the area of interest. Processed images with identified stars (fig. 7) are consistent with the Stellarium sky map.
The Stellarium software was used to compare processed images with a star map. Many stars of magnitude 7 and 8 have been identified on processed images. The weakest identified star was HIP 92005 A (mag. 9.15). The sky map from the Stellarium software is shown in fig. 8 with the area of interest. The ground test of system with compensation of the camera properties and the image enhancement give promising results for using such a low-cost camera system in suborbital missions.

\section{PREPARATION OF MisSION AND EXPECTATION}

The REX experiment has succesfully undergone other required tests, such as a functional test in the vacuum chamber and random vibration test. During the vacuum chamber functional test, the camera system was powered and operated in the same mode as it would be in the space. No overheating problem was observed, even if the duration of the test was much longer than the suborbital mission.

The sensitivity of the camera system is sufficient for mission objectives, able to recognize stars as weak as magnitude 9.15 in the ground tests. In addition, higher sensitivity is expected during the suborbital mission. The image of captured stars in the ground tests scattered on the surface of many pixels due to atmospheric effects and partly due to non-ideal Earth's rotation compensation during the acquisition of time series of images. Also atmospheric attenuation and light pollution of the sky from the nearby city could reduce the sensitivity of the camera. During suborbital mission, captured images will not affect any atmospheric effects and the high quallity attitude control system of sounding rocket minimizes potential blurring of images. The REX experiment was succesfully assembled into a sounding rocket, as shown in fig. 9. The mission is expected to be completed in April 2018.

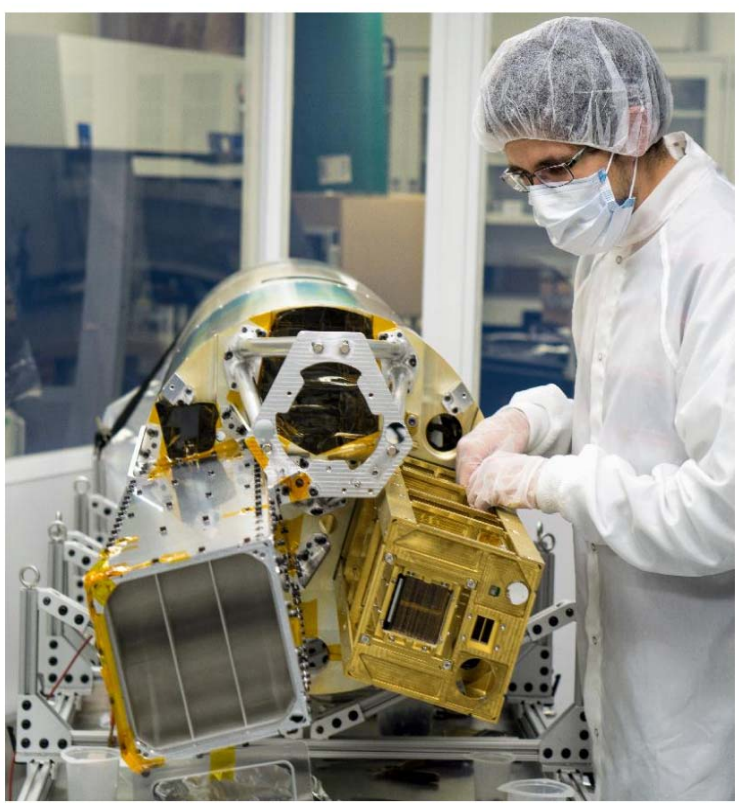

Figure 9. Assembling of REX experiment into sounding rocket. 


\section{CONLUSIONS}

In this paper, we introduced the preparation of the CMOS industrial camera system for the short-term suborbital mission of a sounding rocket as a financial reasonable alternative to expensive space-qualified camera systems. As the ground tests have confirmed, even a small and low-cost industrial camera system with the proper compensation and image postprocessing has sufficient sensitivity to recognize stars of apparent magnitude 9 under the influence of several atmospheric effects. Higher sensitivity is expected during a real mission without the negative effects of the atmosphere. The camera system has successfully passed a vacuum chamber functional test and a random vibration test. However, many aspects of the rocket mission (especially the thermal condition) were not known during the experiment preparation and the true characteristics of the camera system could be evaluated only after the mission data had been obtained. The real mission will take place in April 2018.

\section{ACKNOWLEDGMENT}

This project was funded by Technology Agency of the Czech Republic under project TA04011295: Wideangle X-ray imaging system with Timepix detector.

\section{REFERENCES}

[1] V. Stehlikova, M. Urban, O. Nentvich, A. Inneman, T Dohring, A. C. Probst, "Study of lobster eye optics with iridium coated x-ray mirrors for a rocket experiment" in Proc. SPIE 10235, EUV and X-ray Optics: Synergy between Laboratory and Space. doi: 10.1117/12.2265769.

[2] V. Stehlikova, M. Urban, O. Nentvich, V. Daniel, L. Sieger, J. Tutt, "Hard X-ray Vela supernova observation on rocket experiment WRX-R" in Contributions of the Astronomical Observatory Skalnaté Pleso, vol. 47, no. 2, 2017, Pages 165169, ISSN 1335-1842.

[3] J. P. Carrère, S. Place, J. P. Oddou, D. Benoit, F. Roy, "CMOS image sensor: Process impact on dark current," 2014 IEEE International Reliability Physics Symposium, Waikoloa, HI, 2014, doi: 10.1109/IRPS.2014.6860620. 